\title{
HEMIPARESIA CONGÊNITA
}

\section{AVALIAÇÃO NEUROPSICOLÓGICA COMPLEMENTAR UTILIZANDO COMPUTADOR}

\author{
MARILISA M. GUERREIRO *, MARIA LỨlA G. GARCIA **, \\ GLoria MARIA B. FERRAZ **, ANA MARIA S. G. PIOVESANA *, \\ MARIA VALERIANA L, MOURA-RIBEIRO ***
}

\begin{abstract}
RESUMO - Apresentamos o estudo neuropsicológico de seis crianças portadoras de paralisia cerebral forma hemiplégica, com a ajuda de computador (três crianças com hemiparesia direita e três com hemiparesia esquerda). Os exames tomográficos revelaram cavidades parietais (cisto porencefálico em 4 casos, infarto isquêmico em 1 e cisto subaracnóideo em 1). O objetivo do estudo foi verificar se havia distúrbio na organização espacial, pois os pacientes apresentavam lesão estrutural em áreas parietais e não fora detectado esse distúrbio previamente. Encontramos astereognosia e preferência para o uso da hemi-tela direita do computador nas crianças com hemiparesia direita. Discutimos e propomos explicações para estes achados. Ressaltamos ainda o papel da linguagem computacional Logo, como instrumento adequado para investigação neuropsicológica.
\end{abstract}

PALAVRAS-CHAVE: paralisia cerebral, hemiparesia congênita, neuropsicologia, computador.

Complementary neuropsychological assessment svith computer in congenital hemiparesis.

SUMMARY - We present the neuropsychological assessment with computer aid of six cerebral palsy children. Three children had right hemiparesis and three, left hemiparesis. The tomographic examination showed parietal cavities (porencephalic cyst in 4 children, ischemic injury in 1 case and subarachnoid cyst in 1 case). We have proposed to assess the visuo-spatial function since we suspected the children could have disturbance of this function. We did not detect this disturbance. On the other hand, the children had astereognosia and the right hemiparetic children preferred to execute signs on the right part of the computer visor. We discuss and propose explanations for both findings.

KEY WORDS: cerebral palsy, congenital hemiparesis, neuropsychology, computer.

Luria 10 descreveu três principais unidades funcionais do cérebro, cuja participação conjunta se torna necessária para a atividade mental: Unidade I, que regula a vigília e os estados mentais; Unidade II, que obtém, processa e armazena as informações que chegam do mundo exterior; Unidade III, que programa, regula e verifica a atividade mental. As Unidades II e III podem, por sua vez, ser subdivididas em três zonas corticais de funcionamento (áreas primárias, secundárias e terciárias). As zonas terciárias da unidade funcional II se situam na fronteira entre os córtices occipital, temporal e pós-central, sendo sua maior parte formada pela região parietal inferior, que ocupa quase um quarto da massa

Departamento de Neurologia, Faculdade de Ciências Médicas (FCM), Universidade Estadual de Campinas (UNICAMP): * Professor Assistente da Disciplina de Neurologia Infantil; ** Psicóloga, Pedagoga, Pós-Građuandas em Neurociências; *** Professor Adjunto da Disciplina de Neurologia Infantil. Aceite: 30-março-1993.

Dra. Marilisa M. Guerreiro - Departamento de Neurologia, FCM/UNICAMP - Caixa Postal 6111 - 13081-970 Campinas SP - Brasil. FAX 0192-526773. 
total da segunda unidade funcional. A organização espacial é a principal função das zonas terciárias da unidade funcional II de Luria. Outros autores também salientaram o papel predominante de áreas cerebrais posteriores em tarefas que dependem da organização espacial 1,2,4.

O propósito desta avaliação foi estudar crianças com hemiparesia congênita cujos exames tomográficos apresentavam cavidades parietais (cisto ou atrofia), com o objetivo primordial de verificar se havia distúrbio na organização espacial.

\section{MATERIAL E METODOS}

Foram selecionadas para o presente estudo seis crianças do Ambulatório Especial de Hemiparesia Congenita do Departamento de Neurologia da FCM/UNICAMP.

Observações:

Caso 1. IBS, 5 anos, masculino, branco; quarto filho de casal não consanguineo, a mãe com 39 e o pai com 41 anos por ocasião da gestação não planejada. Houve tentativa de abortamento logo no início, através do uso de medicação endovenosa. $O$ parto foi normal a a termo; o recém-nascido, com peso de $3950 \mathrm{~g}$, não apresentou intercorrencias. O desenvolvimento neuropsicomotor foi considerado normal, mas ao exame neurologico constatou-se síndrome piramidal deficitária e de liberação no hemicorpo esquerdo (F). O eletrencefalograma (EWG) e o raio X simples de crânio (RX) foram normais. A tomografia computadorizada de cranio (TC) revelou agenesia do trígono e septo pelúcido com agenesia parcial do corpo caloso e lesão hipoatenuante frontoparietal direita(D) comunicante com o ventriculo lateral $D$ (cisto porencefálico à $\mathbf{D})$.

Caso 2. ECS, 7 anos, masculino, branco, segundo filho de casal jovem e não consanguíneo. Gestação e parto sem intercorrências. Peso ao nascimento de $2990 \mathrm{~g}$. Houve discreto retardo no desenvolvinento neuropsicomotor. Em relação aos antecedentes apresentava um irmão, mais velho, com diagnóstico de paralisia cerebral e uma irmã, mais nova, falecida com hidranencefalia. Avaliação genética apontou provável interação multifatorial a houve suspeita de citomegalovirose, não confirmada laboratorialmente. O exame neurológico revelou síndrome piramidal deficitária e de liberação à E. O EEG e RX de crânio foram normais. A TC evidenciou agenesia do trígono e septo pelúcido e agenesia parcial do corpo caloso; presença de lesão hipoatenuante frontoparietal $D$ que se comunica com 0 ventrículo lateral $D$ (porencefalia à $D$ ).

Caso 3. AAS, 10 anos, feminina, negra, segunda gestaçã, sendo os pais jovens e não consanguíneos. Houve ganho excessivo de peso, edema e «ameaço» de parto prematuro com uso de medicacão, no oitavo mês da gravidez. Parto cesárea gemelar, choro imediato, sendo o peso do recém-nascido $2250 \mathrm{~g}$. No segundo dia de vida necessitou de incubadora por apresentar dificuldades respiratórias, nela permanecendo durante quinze dias. Sua irmã teve alta com a mãe. O desenvolvimento neuropsicomotor foi normal até os 15 meses, quando passou a apresentar manifestações epilépticas e atraso nas aquisições. $O$ exame neurológico revelou síndrome piramidal deficitária e de liberação à $\mathbf{E}$, atrofia muscular em hemicorpo $\mathbf{E}$ e fala disártrica. A avaliação psicológica evidenciou déficit cognitivo. O EEG foi normal. A TC mostrou dilatação assimétrica dos ventrículos laterais predominando à $D$ e lesão hipoatenuante de limites imprecisos sem efeito de massa em projecão temporoparietal que se comunica com o ventrículo lateral (porencefalia à $D$ e hemiatrofia cerebral D) (Fig. 1).

Caso 4. DRSI, 7 anos, feminina, branca, segunda filha de casal jovem e não consanguíneo. Gestação sem intercorrências. Parto hospitalar, cesárea, sendo o peso 2760g. Apresentou cianose prolongada, permanecendo um dia na incubadora. Seu desenvolvimento neuropsicomotor foi atrasado e ao exame neurológico foi constatada hemiparesia $D$ incompleta de predominio braquial. A avaliação psicológica acusou déficit cognitivo. O EEG mostrou espiculas na região frontotemporal $\mathbf{E}$. A TC evidenciou zona hipoatenuante fronto-parietotemporal $\mathbf{E}$ sugestiva de atrofia localizada (infarto isquêmico).

Caso 5. LCO, 13 anos, feminina, branca, sétima filha de mãe idosa (39 anos); gestação e parto sem intercorrências. Desenvolvimento neuromotor normal, porém com atraso de fala. $O$ exame neurológico mostrou síndrome piramidal deficitária e de liberação à $D$, nistagmo congênito, visão subnormal e estrabismo divergente à D. A avaliação psicológica revelou. déficit cognitivo. O EEG evidenciou atividade lenta contínua no hemisfério cerebral $\mathbf{E}$. $\mathbf{A}$ TC observou-se dỉlatação assimétrica dos ventrículos laterais, predominando à $\mathbf{E}$ e lesão hipoatenuante de limites imprecisos em projeção temporoparietal que se comunica com o ventriculo lateral $\mathrm{E}$ (porencefalia à $\mathbf{E}$ e hemiatrofia cerebral à $\mathbf{E}$ ). 

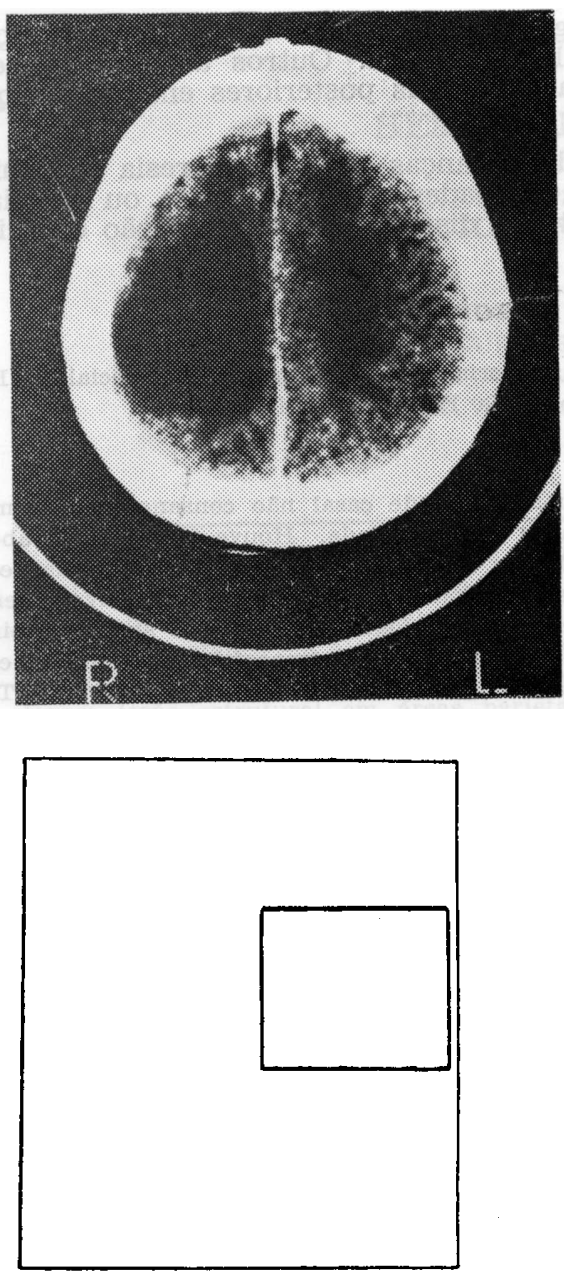

Fig. 2. A figura müstra quadrado à direita da tela. Os pacientes com hemiparesia direita mostraram preferéncia para usar a hemi-tela direita.
Fig. 1. Caso 3. Porencefalia a direita e hemiatrofia cerebral direita.

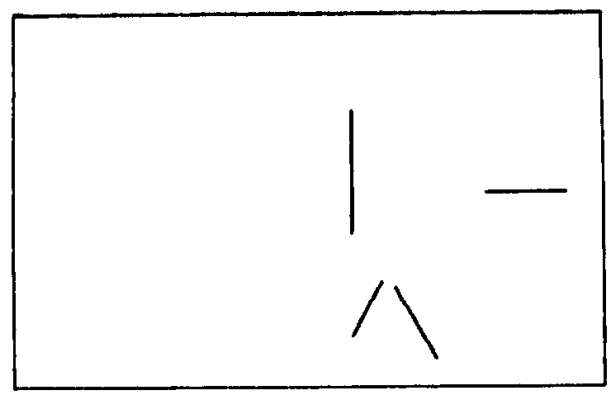

Fig. 3. A figura mostra os quatro tragos à direita da tela, resultados apresentados nos casos 4,5 e 6.

Caso 6. MAS, 11 anos, masculino, branco, segundo filho de casal jovem e năo consangulneo. Gestaçăo e parto transcorreram sem anormalidades, sendo o peso $2700 \mathrm{~g}$. Houve atraso no desenvolvimento neuromotor. O exame neurológico mostrou hemiparesia $D$ incompleta com predomínio braquial. O EWG evidenciou assimetria com aumento do ritmo beta na regiko frontal $\mathbf{E}$, além de espiculas focais máximas na regiăo frontocentral $\mathrm{H}$. A TC mastrou importante atrofia do hemisfério cerebral E, presenca de cisto subaracnóldeo extenso a D, dilataçăo ex-vácuo do ventriculo lateral $\mathrm{E}$ e desvio da linha média para a $\mathrm{F}$.

\section{Metodologia:}

A avallação para investigaçăo de eventual distúrbio espacial constou de:

1. Avaliaçăo neuropsicológica clássica baseada na bateria Luria-Nebraska para crianças 5,6 e avaliaçăo dirigida da funçăo visuo-espacial através de teste proposto por Guerreiro 7. 
2. Avaliação complementar com o uso de computador utilizando a linguagem computacional Logo 11 programada em quatro sessões: a. Introdução de quatro comandos básicos; b. Execução de um quadrado; c. Execução de uma «casa» com moāelo pré-estabelecido; d. Execução de quatro traços simples dispostos em diferentes orientações.

3. Avaliação neuro-oftalmológica enfatizando particularmente o estudo de campo visual.

4. Avaliação da distribuiçăo espacial da atenção, focalizando a avaliaçăo de negligência hemiespacial através da adaptação de teste proposto por Weintrub and Mesulam 15.

\section{RESULTADOS}

A uvaliação neuropsicológica clássica nada revelou além de astereognosia no hemicorpo comprometido. O paciente 1 , com cinco anos de idade teve grande dificuldade em aprender a lidar com o computador devido à baixa idade, ficando sua avaliação prejudicada. Entretanto, realizou testes no papel, em que se observou não haver dificuldade na distribuição espacial de traços.

Os outros pacientes executaram todas as tarefas propostas, tanto no papel quanto no computador. Porém, os pacientes 3,4 e 5 realizaram as tarefas com muita dificuldade, sugerindo comprometimento intelectual.

Todos distribuíram equilibradamente os traços no papel e no computador (exceto o paciente 1) e também apresentaram domínio de ângulos ao realizar quadrados.

Com a execução de testes em computador observamos que houve preferência para se usar a hemi-tela $D$ nos casos 4, 5 e 6 (Figs. 2 e 3), fato também visto discretamente com os testes no papel. Para compiementar o entendimento desse achado realizou-se estudo de campo visual e de negligência hemi-espacial. Não se detectou heminegligência. A avaliação de campo visual revelou-se normal em cinco pacientes. O exame do caso 6 acusou hemianopsia homónima $D$. Assim, os trés pacientes com hemiparesia D mostraram tendência a utilizar preferencialmente a hemi-tela $D$ do computador, inclusive o paciente 6 que apresentava hemianopsia homônima $D$.

\section{COMENTERIOS}

Apesar de todos os pacientes apresentarem evidentes lesões parietais, não detectamos distúrbio espacial através dos testes aplicados e, provavelmente, isto se deve à plasticidade cerebral. Segundo Isabelle Rapin 13, dois mecanismos contam para a recuperação de função em nível celular: regeneração de axónio e remodelação das interconexões sinápticas. Esta autora referiu que neurônios, uma vez «nascidos» não se dividem. Assim, a recuperação não se dá pela restituição de células perdidas. Ocasionalmente ocorre regeneração aberrante e geralmente incompleta, sendo limitada a capacidade de recuperação. Outro mecanismo seria o uso mais ativo de vias alternativas potencialmente utilizáveis, mas até então subsidiárias. A etiologia da hemiparesia em quatro pacientes (casos $1,2,3,5$ ) foi a porencefalia, que se trata de lesão secundária a processo de mediação vascular pré-natal 13 ou decorre de distúrbio de migração neuronal 9. Assim, como as deformidades parietais existentes nos cérebros desses pacientes foram consequentes a alteraçōes precoces, ainda na gestação, possivelmente outras áreas se desen. volveram e adquiriram funções das regiōes comprometidas. O mesmo raciocínio pode ser aplicado ao caso 4 , cujo infarto isquêmico provavelmente decorreu de lesão hipóxico-isquêmica intra-útero. $O$ caso 6 apresentou cisto subaracnóideo tratando-se, portanto, de malformação do sistema nervoso central, também de origem pré-natal, assim não invalidando o raciocínio.

A avaliação neuropsicológica revelou astereognosia como único dado alterado $e$ isto se deve provavelmente a comprometimento das áreas secundárias de Luria 10 referentes ao sistema sensorial. As áreas primárias da segunda unidade funcional de Luria recebem os impulsos provenientes do mundo exterior. A área primária do sistema sensorial corresponde à área 3 de Brodmann. A essas zonas primárias do córtex sensorial geral (parietal) se superpõem as zonas secundárias correspondentes às áreas 1,2 e 5 de Brodmann e parte da área 40 . As áreas primárias, como já comentado, são áreas de projeção. Já as áreas secundárias superpostas são zonas gnósticas, isto é, são as regiōes responsáveis pelo processamento das informaçốes que chegaram às áreas primárias. Lesões dessas áreas 
secundárias levam, portanto, à dificuldade de identificação das informações projetadas no córtex sensorial primário. Como os nossos pacientes apresentam lesóes parietais, entende-se que deve ter havido comprometimento das áreas secundárias do sistema sensorial.

$\mathrm{Na}$ realização de testes em computador, os pacientes com hemiparesia direita mostraram preferência para usar a hemi-tela direita, fato também observado discretamente nos testes em papel. Um desses pacientes (caso 6) apresentava hemianopsia homónima direita. Como a alteração de campo visual na avaliação oftalmológica não explica essa preferência e como também não houve heminegligência, procuramos a justificativa desse achado. Acreditamos que a explicação se encontra na busca visual ("visual search»)12. Assim, o hemisfério esquerdo obtém vantagem na busca visual por causa de sua superioridade analítica, isto é, sua maior capacidade em analisar detalhes. As diferenças de processamento hemisférico são frequentemente caracterizadas como analíticas ou sequenciais para o hemisfério esquerdo e holísticas ou simultâneas para o hemisfério direito 3 . O curioso desse achado é que essa preferência para o uso da hemi-tela direita ocorreu justamente nos três pacientes com lesões em hemisfério esquerdo. Parece ter havido hiperfunção relativa do sistema visual à esquerda como compensação do déficit existente no sistema sensorial.

Foi o uso da linguagem computacional Logo que nos permitiu essas observações, pois no papel as evidências foram discretas. Em estudo realizado previamente 8 pudemos observar que esta linguagem se presta para avaliação espacial e neste estudo pudemos detectar novos dados como fruto do uso do computador e da linguagem Logo na avaliação complementar diagnóstica neuropsicológica.

\section{REFERENCIAS}

1. Benson DF, Barton MI. Disturbances in constructional ability. Cortex 1970, 6:19-46.

2. Benton AL. Disorders of spatial orientation. In: Vinken PJ, Bruyn GW (eds). Handbook of clinical neurology. Amsterdam: North-Holland, 1969, Vol 3, p 212-228.

3. Day PS, Ulatowska HK. Perceptual, cognitive and linguistic development after early hemispherectomy: two case studies. Brain and Language 1979, 7:17-33.

4. De Renzi E. Disorders of spatial orientation. In: Fredericks JAM (ed). Handbook of clinical neurology. Amsterdam: Elsevier, 1985, Vol 1, p 405-422.

5. Golden CJ. The Luria-Nebraska children's battery: theory and formulation. In: Hynd GW, Obrzut JE (eds). Neuropsychological assessment and the school-age child, issues and procedures. New York: Grune \& Stratton, 1981, p 277-302.

6. Golden CJ. The Nebraska neuropsychological children's battery. In: Reynolds CR, Fletcher-Janzen E (eds). Handbook of clinical child neuropsychology. New York: Plenum Press, 1989, p 193-204.

7. Guerreiro MM. Avaliação da função vísuo-espacial em uma criança com paralisia cerebral: proposta de um novo teste. Tese de Mestrado. Faculdade de Ciências Médicas da Universidade Estadual de Campinas. Campinas, 1989.

8. Guerreiro MM, Moura-Ribeiro MVL. Função visuo-espacial em paralisia cerebral e proposta de um novo teste com a ajuda de computador. Arq Neuropsiquiatr 1991, 49:310-314.

9. Jacobson RI. Congenital structural defects. In: Swaiman KF (ed). Pediatric neurology: principles and practice. St. Louis: Mosby, 1989, p 317-362.

10. Luria AR. Higher cortical functions in man. New York: Basic Books, 1966.

11. Papert S. Logo: computadores e educação, São Paulo: Brasiliense, 1985.

12. Polich $\mathfrak{J}$. Hemispheric patterns in visual search. Brain and Cognition 1984, 3:128-139.

13. Rapin I. Brain damage or dysfunction: general considerations. In: Rapin I (ed). Children with brain dysfunction: neurology, cognition, language and behavior. New York: Raven Press, 1982, p 23-34.

14. Rosemberg $\mathbf{S}$. Das encefalopatias circulatórias pré e perinatais: estudo neuropatológico. Suas Implicaçōes Patogênicas. Tese de Doutoramento. Pontificia Universidade Católica de São Paulo. Săo Paulo, 1974.

15. Weintraub SO, Mesulam M-M. Mental state assessment of young and elderly adults in behavioral neurology. In: Mesulam M-M (ed). Principles of behavioral neurology. Phidelphia: Davis, 1985, p 71-123. 